\title{
HIV-1 predisposed to acquiring resistance to maraviroc (MVC) and other CCR5 antagonists in vitro has an inherent, low-level ability to utilize MVC-bound CCR5 for entry
}

Michael Roche ${ }^{1,2}$, Martin R Jakobsen ${ }^{1,3}$, Anne Ellett ${ }^{1}$, Hamid Salimiseyedabad ${ }^{1}$, Becky Jubb ${ }^{4}$, Mike Westby ${ }^{4}$, Benhur Lee ${ }^{5}$, Sharon R Lewin ${ }^{1,2,6}$, Melissa J Churchill ${ }^{1,7}$ and Paul R Gorry ${ }^{1,2,8^{*}}$

\begin{abstract}
Background: Maraviroc (MVC) and other CCR5 antagonists are HIV-1 entry inhibitors that bind to- and alter the conformation of CCR5, such that CCR5 is no longer recognized by the viral gp120 envelope (Env) glycoproteins. Resistance to CCR5 antagonists results from HIV-1 Env acquiring the ability to utilize the drug-bound conformation of CCR5. Selecting for HIV-1 resistance to CCR5-antagonists in vitro is relatively difficult. However, the CCR5-using CC1/85 strain appears to be uniquely predisposed to acquiring resistance to several CCR5 antagonists in vitro including MVC, vicriviroc and AD101.

Findings: Here, we show that Env derived from the parental CC1/85 strain is inherently capable of a low affinity interaction with MVC-bound CCR5. However, this phenotype was only revealed in 293-Affinofile cells and NP2-CD4/ CCR5 cells that express very high levels of CCR5, and was masked in TZM-bl, JC53 and U87-CD4/CCR5 cells as well as PBMC, which express comparatively lower levels of CCR5 and which are more commonly used to detect resistance to CCR5 antagonists.

Conclusions: Env derived from the CC1/85 strain of HIV-1 is inherently capable of a low-affinity interaction with MVC-bound CCR5, which helps explain the relative ease in which CC1/85 can acquire resistance to CCR5 antagonists in vitro. The detection of similar phenotypes in patients may identify those who could be at higher risk of virological failure on MVC.
\end{abstract}

\section{Introduction}

Human immunodeficiency virus type 1 (HIV-1) entry is initiated by the interaction of the viral gp120 envelope (Env) glycoproteins with cellular CD4 and a coreceptor, either CCR5 or CXCR4 [1]. Maraviroc (MVC) and other CCR5-antagonists such as vicriviroc (VVC, also known as SCH-D), AD101 (a preclinical precursor of VVC), and aplaviroc (APL) are HIV-1 entry inhibitors that bind toand alter the conformation of CCR5, such that CCR5 is no longer recognized by gp120 [1]. Thus, CCR5-antagonists are allosteric inhibitors of HIV-1 entry [2-4]. MVC has been approved for use in treatment-experienced and

\footnotetext{
* Correspondence: gorry@burnet.edu.au

${ }^{1}$ Center for Virology, Burnet Institute, Melbourne, Victoria, Australia Full list of author information is available at the end of the article
}

antiretroviral therapy (ART)-naïve HIV-1-infected adults who have no evidence of CXCR4-using virus in plasma [5]. As with other antiretrovirals, treatment with CCR5antagonists can result in drug resistance leading to virological rebound. Although virological failure can arise from the emergence of CXCR4-using HIV-1 strains that were present at very low levels prior to initiation of a CCR5-antagonist [6], genuine resistance to CCR5antagonists results from adaptive alterations in gp120 enabling recognition of the drug-bound conformation of CCR5 [7-15].

Being allosteric inhibitors of virus entry, resistance to CCR5-antagonists is evident by plateaus in virus inhibition curves below $100 \%$ inhibition [16]. The magnitude of the reduction in plateau height can be quantified as the maximal percent inhibition (MPI), which reflects the 
ability of HIV-1 gp120 to recognize the drug bound conformation of CCR5. For example, MPIs can be high (> 80\%) [15] signifying a relatively inefficient ability of gp120 to utilize the drug-bound conformation of CCR5, or low $(<20 \%)$ [13] signifying relatively efficient utilization of drug-bound CCR5. However, MPIs can be influenced by differences in the level of CCR 5 expression on target cell populations $[9,11,12]$. Generally, in cell lines, there is an inverse relationship between the MPI achieved by a given virus with resistance to a CCR5-antagonist, and the level of CCR5 expression. Clinically, MPIs of HIV-1 have been reported using the PhenoSense ${ }^{\mathrm{TM}}$ Entry assay [16], which uses the U87-CD4/CCR5 cell line. These cells express comparatively lower levels of CCR 5 than other commonly used indicator cells such as TZMbl, JC53 and NP2-CD4/CCR5 cells [12] and therefore, are likely to provide a relatively conservative measure of resistance to CCR5-antagonists. Consistent with this view, results from the clinical trials of MVC in treatment-experienced subjects (MOTIVATE) showed that most MVC-resistant viruses in subjects failing therapy had relatively high MPIs within the range of $80-95 \%$, when tested using the PhenoSense ${ }^{\mathrm{TM}}$ Entry assay ([15] and references within).

The in vitro generation and characterization of HIV-1 variants with resistance to antiretroviral drugs is vital for elucidating resistance mechanisms. However, selecting for HIV-1 resistance to CCR5-antagonists is relatively difficult [16]. One particular HIV-1 strain, CC1/85 [17], has been used in a number of independent studies for the in vitro generation of HIV-1 resistance to different CCR5-antagonists including MVC, VVC and AD101 (for example, $[16,18-20])$. In fact, the published in vitro CCR5-antagonist resistance studies are heavily biased towards the characterization of resistant variants derived from $\mathrm{CC} 1 / 85$. The $\mathrm{CC} 1 / 85$ strain of HIV-1 may therefore be predisposed to acquiring resistance to CCR5- antagonists in vitro. Here, we sought to elucidate the phenotypic features of $\mathrm{CC} 1 / 85$ that underlie this predisposition. A better understanding of these mechanisms has the potential to identify subjects with increased risk of developing resistance to MVC and other CCR5-antagonists.

\section{Methods}

MVC-Sens and MVC-Res plasmids contain the env gene of $\mathrm{CC} 1 / 85$ virus and a derivative with MVC-resistance, respectively, cloned into the pSVIII-Env expression vector $[15,16]$. Single-round luciferase reporter viruses pseudotyped with MVC-Senv or MVC-Res Envs, or with the CCR5-using (R5) YU2, JRCSF, NB6-C3 or NB8-C4 Envs as controls were produced as described previously [15]. The characterization and maintenance of TZM-bl, JC53, U87-CD4/CCR5, NP2-CD4/CCR5 and the dually CD4- and CCR5-inducible 293-Affinofile cells, and the preparation of peripheral blood mononuclear cells (PBMC) has been described previously [15,21]. Maraviroc resistance assays were conducted using Env-pseudotyped luciferase reporter viruses, or replication competent viruses carrying MVC-Res or MVC-Sens env genes, as described previously $[15,16]$. For experiments using 293Affinofile cells, populations expressing CD4 together with different levels of CCR5 ranging from relatively low to high were generated by inducing the cells with 2.5 or $5 \mathrm{ng}$ per $\mathrm{ml}$ of minocycline and either 15.6, 31.2, 62.5, $125,250,500,1000$ or $2000 \mathrm{nM}$ of ponasterone A, as described previously [21]. Alterations in drug sensitivity were assessed by reductions in the MPI as described previously $[15,16]$.

\section{Results}

As part of our ongoing studies of HIV-1 resistance to MVC and other CCR5-antagonists [15], in particular the influence of cell-surface CCR5 levels on the ability of resistant viruses to recognize the drug-bound conformation of CCR5, we first conducted MVC resistance assays for MVC-Res and MVC-Sens Envs in four different cell lines that express varying levels of CCR5, as well as PBMC. The cell lines included U87-CD4/CCR5 cells which express comparatively low-levels of CCR5, TZM-bl and JC53 cells which express comparatively moderate levels of CCR5, and NP2-CD4/CCR5 cells which express comparatively high levels of CCR5 (Figure 1A). The MPIs for MVC-Res Env ranged from approximately 55\% in NP2-CD4/CCR5 cells to as high as $97 \%$ in U87-CD4/CCR5 cells (Figure 1B). Therefore, consistent with previous studies of VVCand APL-resistant HIV-1 [11,12], we observed a close inverse relationship between CCR5 expression levels on cell lines and the magnitude of the MPI for MVC-Res Env. However, the most pronounced phenotypic resistance to MVC was in PBMC (Figure 2), which express much lower levels of CCR5, typically in the order of 5,000 to 12,000 molecules of CCR5 per CD4+/CCR5+ T-lymphocyte depending on the donor [22]. These results are consistent with those of previous studies, which suggest that distinct forms of CCR5 may exist on primary cells and cell lines that have varying affinities for CCR5 antagonists [23]. Unexpectedly, while virus pseudotyped with MVC-Sens Env was completely inhibited by MVC in U87-CD4/ CCR5, TZM-bl and JC53 cells (Figure 1B) and PBMC (Figure 2A), this virus was consistently incompletely inhibited in NP2-CD4/CCR5 cells, plateauing at approximately $96 \%$ inhibition at the highest concentrations of MVC (Figure 1B). Interestingly, incomplete inhibition of Env derived from $\mathrm{CC} 1 / 85$ to MVC and another CCR5 antagonist, $\mathrm{SCH}-\mathrm{C}$ has also been noted in two previous studies using different experimental approaches [16,24]. In contrast to the incomplete inhibition of virus pseudotyped with MVC-Sens Env in NP2-CD4/CCR5 cells, viruses 


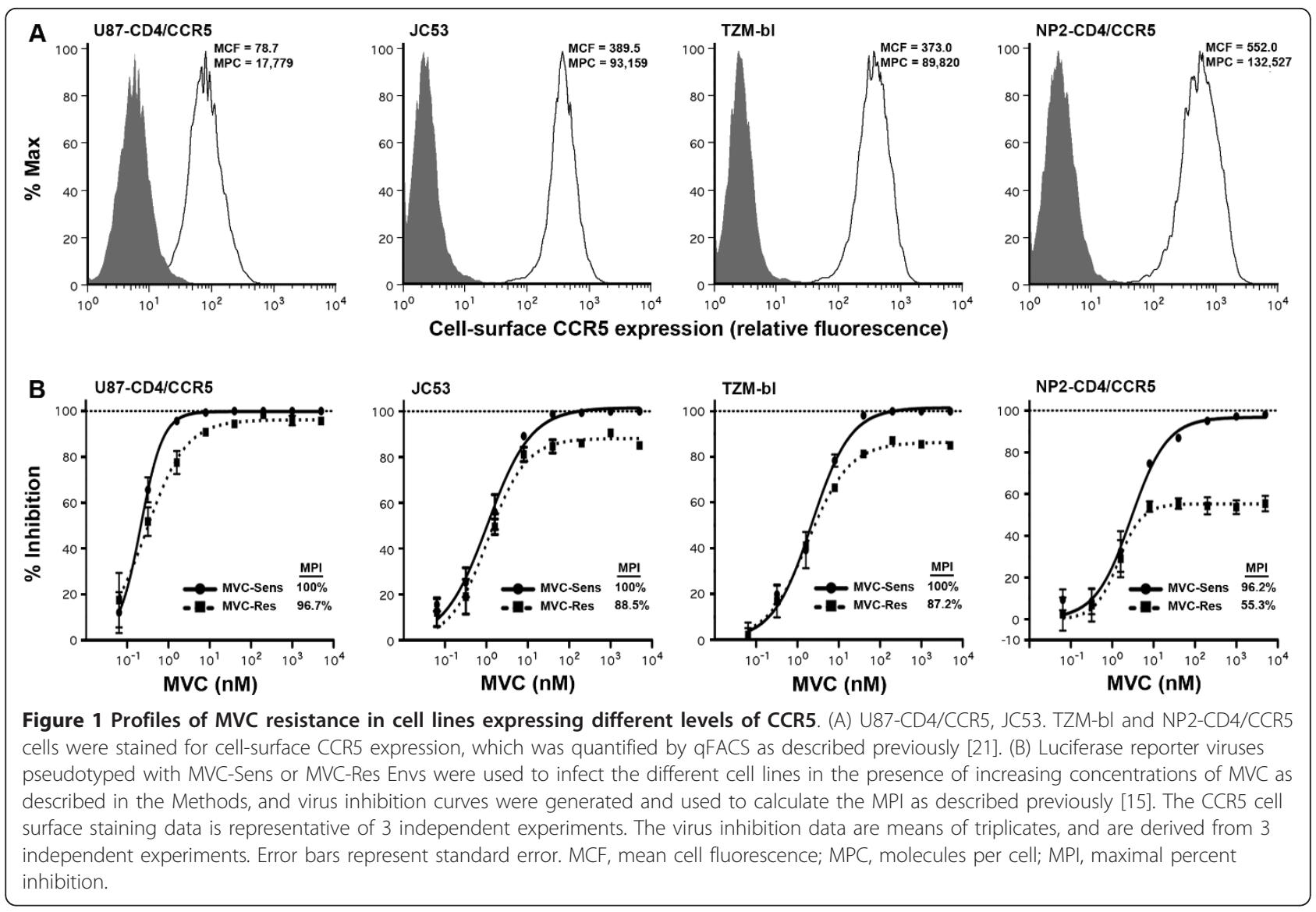

pseudotyped with control YU2, JRCSF, NB6-C3 and NB8C4 Envs were completely inhibited by MVC in these cells with MPIs consistently at $100 \%$ (data not shown). Together, these results show that MVC-Sens Env maintains a MVC-sensitive phenotype in PBMC and cell lines expressing relatively low or moderate CCR5 levels, including U87-CD4/CCR5 cells that are used in the PhenoSense ${ }^{\mathrm{TM}}$ Entry assay, but displays a low-level of basal resistance to MVC in NP2-CD4/CCR5 cells that express considerably higher levels of CCR5.

To more precisely determine the relationship between CCR5 expression levels and the detection of this phenotype of MVC-Sens Env, we next performed MVC inhibition assays in 293-Affinofile cells where we could tightly control the induction of CCR5 in a uniform cell type. 293Affinofile cells were induced to express CD4 together with eight different levels of CCR5 (ranging from approximately 8,000 to 167,000 molecules of CCR5 per cell) (Figure 3B) and subjected to entry assays with luciferase reporter viruses pseudotyped with MVC-Sens or MVC-Res Envs in the presence or absence of $10 \mu \mathrm{M}$ MVC (Figure 3A). In the absence of drug, we observed a similar pattern of virus entry levels between MVC-Sens and MVC-Res Envs in all cell populations, indicating a similar CCR5-dependence profile. In the presence of drug, as expected [15], MVCRes Env entered cells efficiently, particularly when cells were expressing high levels of CCR5. MVC-Sens Env entered cells expressing relatively high levels of CCR 5 in the presence of drug, albeit less efficiently than MVC-Res Env, but was completely inhibited by MVC in cells expressing comparatively moderate and low-levels of CCR5. In contrast, luciferase reporter viruses pseudotyped with control YU2, JRCSF, NB6-C3 and NB8-C4 Envs were completely inhibited by MVC in 293-Affinofile cells expressing the highest levels of CCR5 (Figure 3C). Together, these results confirm the observations in NP2-CD4/CCR5 cells (Figure 1B) that MVC-Sens Env displays a low-level MVC-resistant phenotype that is revealed only when cells are expressing high levels of CCR5, but masked when cells are expressing moderate or relatively low-levels of CCR5.

To determine whether this apparent low-level basal resistance could be due to an inherent ability of MVCSens Env to recognize drug-modified CCR5, we next produced virus inhibition curves for MVC-Sens and MVCRes Envs in differentially induced 293-Affinofile cell populations (Figure 4). MVC-Res Env achieved plateaus of incomplete inhibition by MVC in all cell populations, with MPIs ranging from approximately 20 to $40 \%$ in a CCR 5 


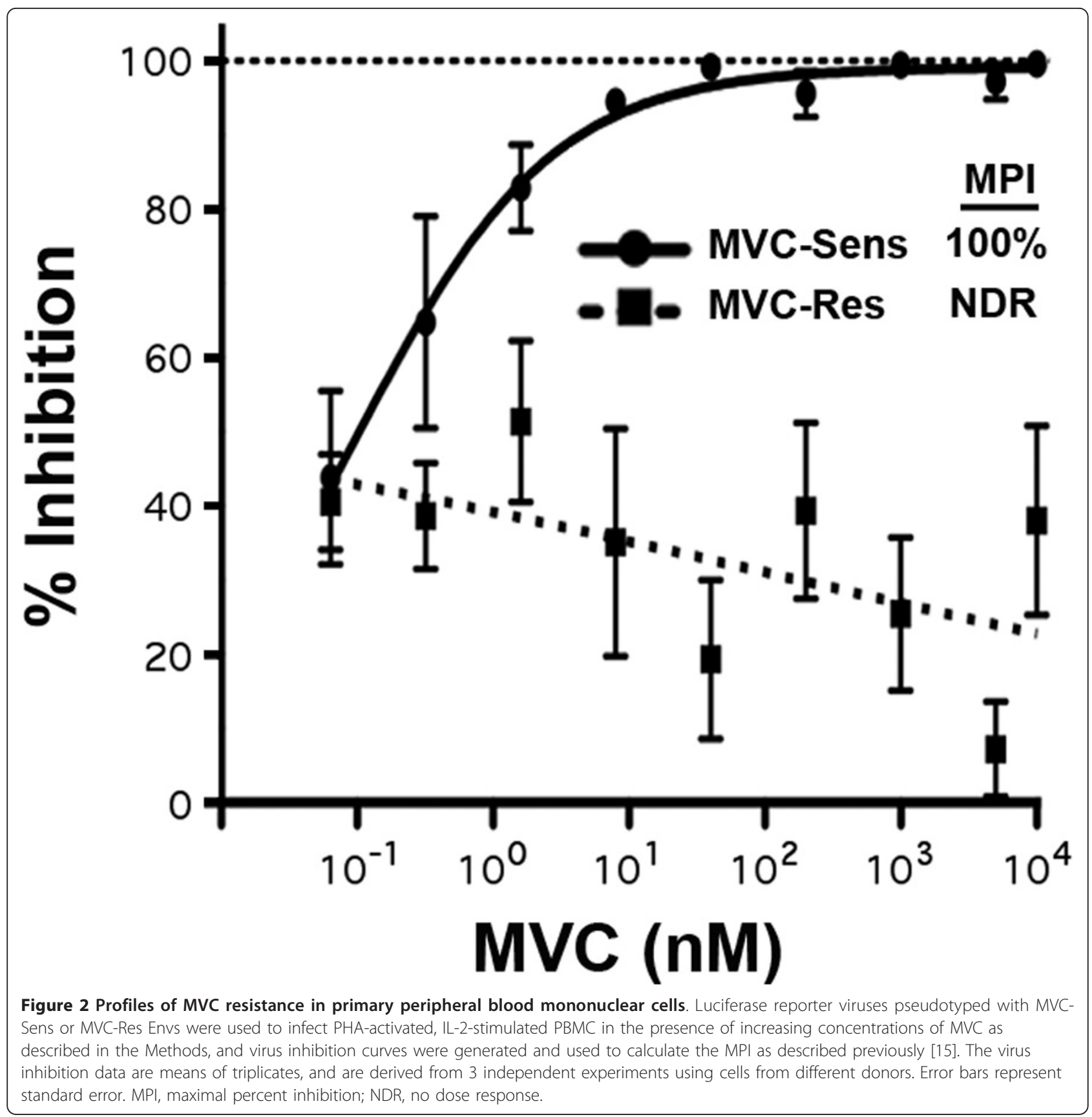

concentration-dependent manner. MVC-Sens Env was completely inhibited by MVC in cells expressing low-levels of CCR5, but achieved plateaus of incomplete inhibition in cells expressing higher levels of CCR5, with MPIs ranging from approximately 53 to $100 \%$ in a CCR 5 concentrationdependent manner. In contrast, viruses pseudotyped with control YU2 and JRCSF Envs achieved plateaus of 100\% inhibition in all cell populations, despite a clear association between the MVC $\mathrm{IC}_{50}$ and CCR5 expression levels. Whilst the association between the $\mathrm{MVC} \mathrm{IC}_{50}$ for $\mathrm{YU} 2$ and JRCSF and CCR5 expression levels suggests that more
MVC is required to achieve complete inhibition of these viruses as CCR5 levels are increased, the plateaus of incomplete inhibition by MVC-Sens Env are consistent with the interpretation that it possesses the ability to interact with the drug-bound conformation of CCR5 [16]. However, the fact that this occurs only in cells expressing high levels of CCR5 supports the interpretation that this is a low affinity interaction [11]. Together, these results suggest that MVC-Sens Env can be distinguished from other R5 Envs by an inherent, yet relatively inefficient capability of recognizing the MVC-bound conformation of CCR5. 

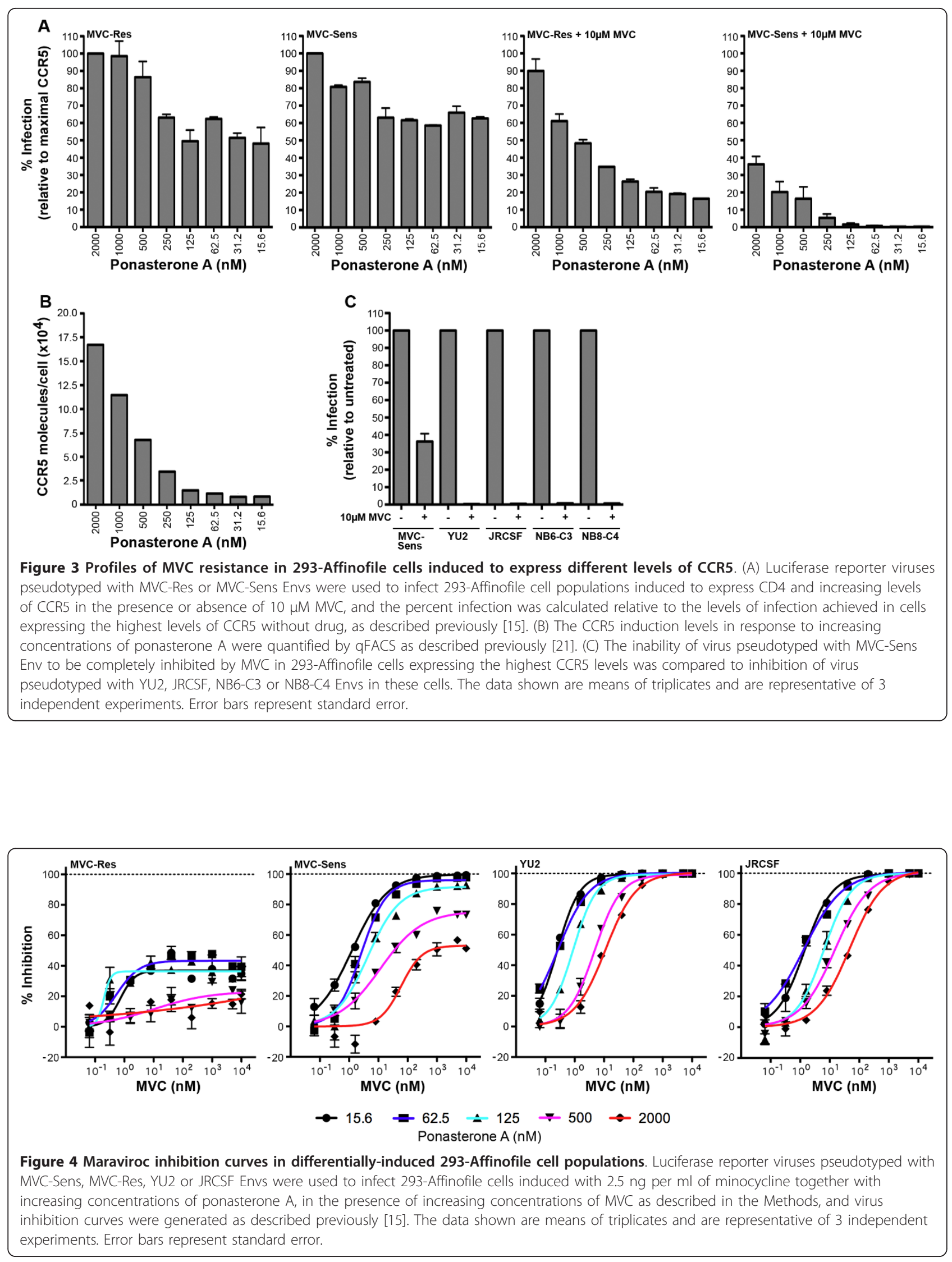


\section{Discussion and conclusions}

Our results suggest that MVC-Sens Env, which was derived from the CC1/85 strain of HIV-1, has an inherent ability to recognize the MVC-modified conformation of CCR5. These results provide a plausible explanation as to why $\mathrm{CC} 1 / 85$, but not most other R5 HIV-1 strains, appears to be predisposed to acquiring resistance to MVC and other CCR5-antagonists in vitro, and why $\mathrm{CC} 1 / 85$ has been frequently used for this purpose in a number of independent studies [16,18-20]. The disclosure of the ability of MVC-Sens Env, but not other R5 Envs, to recognize drugbound CCR5 in cell lines engineered to overexpress CCR5 distinguishes $\mathrm{CC} 1 / 85$ from other $\mathrm{R} 5$ viruses as being predisposed to develop a genuinely resistant profile when cultured in the presence of increasing concentrations of CCR5-antagonist. However, despite this predisposition, 17 virus passages and multiple Env mutations were required for $\mathrm{CC} 1 / 85$ to acquire resistance to $\mathrm{MVC}$, compared to only 1 passage for this virus to acquire resistance to $3 \mathrm{TC}$ [16], suggesting that even $\mathrm{CC} 1 / 85$ has a relatively high genetic barrier to acquiring resistance to CCR5antagonists.

The results of this study suggest that a similar baseline ability of HIV-1 to interact with drug-modified CCR5 may exist in certain subjects prior to commencing MVC or other CCR5-antagonists. To this end, in a longitudinal study of 21 ART-naïve subjects with HIV-1 subtype C, we have identified two subjects whose viruses exhibit plateaus of incomplete inhibition by MVC in NP2-CD4/CCR5 cells and 293-Affinofile cells expressing high levels of CCR5, but which are completely inhibited by MVC in cell lines expressing lower levels of CCR5, in a strikingly similar fashion as CC1/85 ([25], manuscript in preparation). In addition, retrospective analysis of 11 subjects who developed APL-resistance during the CCR100136 (EPIC) clinical trial of APL showed that baseline viruses of 8 individuals (73\%) had some evidence of partial APL-resistance prior to therapy [8]. Further analysis of one of these baseline viruses confirmed that the Env glycoproteins had a low-affinity interaction with APL-bound CCR5 [12]. The clinical significance of viral variants with low-level basal recognition of drug-bound CCR5 in the setting of MVC and other CCR5antagonist therapies remains to be determined by more extensive in vivo studies. To this end, we have shown that the likelihood of developing resistance to CCR5-antagonists in vivo is influenced also by the activity of the patient's optimized background therapy (B. Jubb and M. Westby, unpublished data). Nonetheless, our results suggest that certain individuals could be at increased risk of drug failure on MVC and other CCR5-antagonists due to predisposition for development of resistance.

One reason why U87-CD4/CCR5 cells are justifiably used by the PhenoSense ${ }^{\mathrm{TM}}$ Entry assay for the measurement of HIV-1 resistance to CCR5-antagonists is because the
CCR5 expression levels on these cells more closely reflects CCR5 levels on primary CD4+ T-cells [22]. However, should future in vivo studies demonstrate that patients with baseline HIV-1 strains possessing inherent low-level ability to recognize drug-bound CCR5 are at greater risk of drug failure due to a predisposition to develop resistance, this phenotype is likely to be masked or only weakly exposed in U87-CD4/CCR5 cells. Pre-screening candidates for CCR5-antagonist therapy by a modified drug susceptibility assay using NP2-CD4/CCR5 cells or 293-Affinofile cells could potentially identify these individuals.

\section{Acknowledgements and funding}

We thank J. Sodroski for providing PSVIII-YU2 Env plasmid and for providing pCMV $\triangle$ P1 $1 \triangle$ envpA and pHIV-1Luc plasmids. We also thank D. Kabat for providing JC53 cells, N. Shimizu and H. Hoshino for permission to use NP2CD4/CCR5 cells, and D. Mosier and R. Nedellec for supplying the NP2-CD4/ CCR5 cells. The following reagents were obtained through the NIH AIDS Research and Reference Reagent Program, Division of AIDS, NIAID, NIH: TZM-bl cells from Dr. John C. Kappes, Dr. Xiaoyun Wu and Tranzyme Inc;; U87-CD4/ CCR5 cells from Dr. HongKui Deng and Dr. Dan R. Littman.

This study was supported by a grant by the Australian Center for HIV and Hepatitis Virology Research (ACH2) to PRG and MJC, and by a grant from NIH/ NIAID to BL (R21 Al092218). MR is supported by a Monash University Postgraduate Research Scholarship. PRG is the recipient of an Australian National Health and Medical Research Council (NHMRC) Level 2 Biomedical Career Development Award. SRL is the recipient of a NHMRC Practitioner Fellowship. The authors gratefully acknowledge the contribution to this work of the Victorian Operational Infrastructure Support Program received by the Burnet Institute.

\section{Author details}

${ }^{1}$ Center for Virology, Burnet Institute, Melbourne, Victoria, Australia. ${ }^{2}$ Department of Medicine, Monash University, Melbourne, Victoria, Australia. ${ }^{3}$ Department of Medical Microbiology and Immunology, Aarhus University, Denmark. ${ }^{4}$ Pfizer Global Research and Development, Sandwich, UK. ${ }^{5}$ Department of Microbiology, Immunology and Molecular Genetics, David Geffen School of Medicine, UCLA, Los Angeles, CA, USA. ${ }^{\circ}$ Infectious Diseases Unit, Alfred Hospital, Melbourne, Victoria, Australia. ${ }^{7}$ Department of Microbiology, Monash University, Melbourne, Victoria, Australia. ${ }^{8}$ Department of Microbiology and Immunology, University of Melbourne, Parkville, Victoria, Australia.

\section{Authors' contributions}

$M R, M R J$ and PRG designed the experiments. MR, MRJ, AE and HS performed the experiments. BJ, MW and BL supplied critical reagents and helped interpret the results. SRL and MJC helped interpret the results. PRG and MJC supervised the project. MR and PRG wrote the manuscript. All authors helped edit the manuscript and have read and approved the final version.

\section{Competing interests}

BJ and MW are employed by Pfizer Global Research and Development. PRG and SRL are members of the ViiV Australia Scientific Advisory Board, and have received honoraria. SRL has received honoraria from ViiV for travel to conferences as well as for speaking at and Chairing ViiV-sponsored events. The other authors declare that they have no competing interests.

Received: 25 August 2011 Accepted: 7 November 2011

Published: 7 November 2011

\section{References}

1. Gorry PR, Ancuta P: Coreceptors and HIV-1 pathogenesis. Current HIV/AIDS Reports 2011, 8:45-53.

2. Dragic T, Trkola A, Thompson DA, Cormier EG, Kajumo FA, Maxwell E, Lin SW, Ying W, Smith SO, Sakmar TP, Moore JP: A binding pocket for a small molecule inhibitor of HIV-1 entry within the transmembrane helices of CCR5. Proc Natl Acad Sci USA 2000, 97:5639-5644. 
3. Seibert C, Ying W, Gavrilov S, Tsamis F, Kuhmann SE, Palani A, Tagat JR, Clader JW, McCombie SW, Baroudy BM, et al: Interaction of small molecule inhibitors of HIV-1 entry with CCR5. Virology 2006, 349:41-54.

4. Tsamis F, Gavrilov S, Kajumo F, Seibert C, Kuhmann S, Ketas T, Trkola A, Palani A, Clader JW, Tagat JR, et al: Analysis of the mechanism by which the small-molecule CCR5 antagonists SCH-351125 and SCH-350581 inhibit human immunodeficiency virus type 1 entry. J Virol 2003, 77:5201-5208.

5. Gorry PR, Ellett A, Lewin SR: Maraviroc. In Kucers' The Use of Antibiotics.. 6 edition. Edited by: Grayson L, Crowe S, McCarthy J, Mills J, Mouton J, Norrby SR, Paterson D, Pfaller M. London: Hodder 2010:2869-2876.

6. Westby M, Lewis M, Whitcomb J, Youle M, Pozniak AL, James IT, Jenkins TM, Perros $M$, van der Ryst E: Emergence of CXCR4-using human immunodeficiency virus type 1 (HIV-1) variants in a minority of HIV-1infected patients following treatment with the CCR5 antagonist maraviroc is from a pretreatment CXCR4-using virus reservoir. I Virol 2006, 80:4909-4920.

7. Berro R, Sanders RW, Lu M, Klasse PJ, Moore JP: Two HIV-1 variants resistant to small molecule CCR5 inhibitors differ in how they use CCR5 for entry. PLoS Pathog 2009, 5:e1000548.

8. Kitrinos KM, Amrine-Madsen H, Irlbeck DM, Word JM, Demarest JF: Virologic failure in therapy-naive subjects on aplaviroc plus lopinavir-ritonavir: detection of aplaviroc resistance requires clonal analysis of envelope. Antimicrob Agents Chemother 2009, 53:1124-1131.

9. Pfaff JM, Wilen CB, Harrison JE, Demarest JF, Lee B, Doms RW, Tilton JC: HIV-1 resistance to CCR5 antagonists associated with highly efficient use of CCR5 and altered tropism on primary CD4+ T cells. J Virol 2010, 84:6505-6514

10. Pugach P, Marozsan AJ, Ketas TJ, Landes EL, Moore JP, Kuhmann SE: HIV-1 clones resistant to a small molecule CCR5 inhibitor use the inhibitorbound form of CCR5 for entry. Virology 2007, 361:212-228.

11. Pugach P, Ray N, Klasse PJ, Ketas TJ, Michael E, Doms RW, Lee B, Moore JP: Inefficient entry of vicriviroc-resistant HIV-1 via the inhibitor-CCR5 complex at low cell surface CCR5 densities. Virology 2009, 387:296-302.

12. Tilton JC, Amrine-Madsen $H$, Miamidian JL, Kitrinos KM, Pfaff J, Demarest JF, Ray N, Jeffrey JL, Labranche CC, Doms RW: HIV type 1 from a patient with baseline resistance to CCR5 antagonists uses drug-bound receptor for entry. AIDS Res Hum Retroviruses 2010, 26:13-24.

13. Tilton JC, Wilen CB, Didigu CA, Sinha R, Harrison JE, Agrawal-Gamse C, Henning EA, Bushman FD, Martin JN, Deeks SG, Doms RW: A maravirocresistant HIV-1 with narrow cross-resistance to other CCR5 antagonists depends on both $\mathrm{N}$-terminal and extracellular loop domains of drugbound CCR5. J Virol 2010, 84:10863-10876.

14. Ogert RA, Hou Y, Ba L, Wojcik L, Qiu P, Murgolo N, Duca J, Dunkle LM, Ralston R, Howe JA: Clinical resistance to vicriviroc through adaptive V3 loop mutations in HIV-1 subtype D gp120 that alter interactions with the N-terminus and ECL2 of CCR5. Virology 2010, 400:145-155.

15. Roche M, Jakobsen MR, Sterjovski J, Ellett A, Posta F, Lee B, Jubb B, Westby M, Lewin SR, Ramsland PA, et al: HIV-1 escape from the CCR5 antagonist maraviroc associated with an altered and less efficient mechanism of gp120-CCR5 engagement that attenuates macrophagetropism. J Virol 2011, 85:4330-4342.

16. Westby M, Smith-Burchnell C, Mori J, Lewis M, Mosley M, Stockdale M, Dorr P, Ciaramella G, Perros M: Reduced maximal inhibition in phenotypic susceptibility assays indicates that viral strains resistant to the CCR5 antagonist maraviroc utilize inhibitor-bound receptor for entry. J Virol 2007, 81:2359-2371.

17. Connor Rl, Sheridan KE, Ceradini D, Choe S, Landau NR: Change in coreceptor use coreceptor use correlates with disease progression in HIV-1-infected individuals. J Exp Med 1997, 185:621-628.

18. Marozsan AJ, Kuhmann SE, Morgan T, Herrera C, Rivera-Troche E, Xu S, Baroudy BM, Strizki J, Moore JP: Generation and properties of a human immunodeficiency virus type 1 isolate resistant to the small molecule CCR5 inhibitor, SCH-417690 (SCH-D). Virology 2005, 338:182-199.

19. Pugach $P$, Ketas TJ, Michael E, Moore JP: Neutralizing antibody and antiretroviral drug sensitivities of HIV-1 isolates resistant to small molecule CCR5 inhibitors. Virology 2008, 377:401-407.

20. Trkola A, Kuhmann SE, Strizki JM, Maxwell E, Ketas T, Morgan T, Pugach P, Xu S, Wojcik L, Tagat J, et al: HIV-1 escape from a small molecule, CCR5specific entry inhibitor does not involve CXCR4 use. Procedings of the National Academy of Sciences USA 2002, 99:395-400.
21. Johnston SH, Lobriz MA, Nguyen S, Lassen K, Delair S, Posta F, Bryson YJ, Arts EJ, Chou T, Lee B: A quantitative affinity-profiling system that reveals distinct CD4/CCR5 usage patterns among human immunodeficiency virus type 1 and simian immunodeficiency virus strains. Journal of Virology 2009, 83:11016-11026.

22. Lee B, Sharron M, Montaner LJ, Weissman D, Doms RW: Quantification of CD4, CCR5, and CXCR4 levels on lymphocyte subsets, dendritic cells, and differentially conditioned monocyte-derived macrophages. Proc Natl Acad Sci USA 1999, 96:5215-5220.

23. Anastassopoulou CG, Ketas TJ, Klasse PJ, Moore JP: Resistance to CCR5 inhibitors caused by sequence changes in the fusion peptide of HIV-1 gp41. Proc Natl Acad Sci USA 2009, 106:5318-5323.

24. Kuhmann SE, Pugach P, Kunstman KJ, Taylor J, Stanfield RL, Snyder A, Strizki JM, Riley J, Baroudy BM, Wilson IA, et al: Genetic and phenotypic analyses of human immunodeficiency virus type 1 escape from a smallmolecule CCR5 inhibitor. J Virol 2004, 78:2790-2807.

25. Jakobsen MR, Roche M, Sterjovski J, Ellett A, Posta F, Lee B, Churchill MJ, Gorry PR: Characterisation of the virus-cell interactions by HIV-1 subtype $C$ variants from an antiretroviral therapy-naive subject with baseline resistance to the CCR5 inhibitor maraviroc. Program and Abstracts of the Keystone Symposium on HIV Evolution, Genomics and Pathogenesis, Whistler 2011, March 20-25, poster 248.

doi:10.1186/1742-4690-8-89

Cite this article as: Roche et al:: HIV-1 predisposed to acquiring resistance to maraviroc (MVC) and other CCR5 antagonists in vitro has an inherent, low-level ability to utilize MVC-bound CCR5 for entry. Retrovirology 2011 8:89.

\section{Submit your next manuscript to BioMed Central and take full advantage of:}

- Convenient online submission

- Thorough peer review

- No space constraints or color figure charges

- Immediate publication on acceptance

- Inclusion in PubMed, CAS, Scopus and Google Scholar

- Research which is freely available for redistribution

Submit your manuscript at www.biomedcentral.com/submit
C Biomed Central 